\title{
SOLVING EQUATIONS OF LENGTH SEVEN OVER TORSION-FREE GROUPS
}

\author{
Mairaj Bibi \\ Department of Mathematics \\ Comsats Institute of Information Technology \\ Park Road, Islamabad 45550, Pakistan \\ e-mail:mairaj_maths@comsats.edu.pk
}

\author{
Martin Edjvet \\ School of Mathematical Sciences \\ The University of Nottingham \\ University Park, Nottingham NG7 2RD, UK \\ e-mail:martin.edjvet@nottingham.ac.uk
}

\begin{abstract}
Prishchepov [16] proved that all equations of length at most six over torsion-free groups are solvable. A different proof was given by Ivanov and Klyachko in [12]. This supports the conjecture stated by Levin [15] that any equation over a torsion-free group is solvable. Here it is shown that all equations of length seven over torsion-free groups are solvable.
\end{abstract}

2000 Mathematics Subject Classification: 20F06

\section{Introduction}

Let $G$ be a non-trivial group and $t$ an element distinct from $G$. An equation (in $t$ ) over $G$ of length $n$ is an expression of the form

$$
s(t)=g_{1} t^{\varepsilon_{1}} g_{2} t^{\varepsilon_{2}} \ldots g_{n} t^{\varepsilon_{n}} \quad\left(g_{i} \in G, \varepsilon_{i}= \pm 1\right)
$$

in which it is assumed that $\varepsilon_{i}+\varepsilon_{i+1}=0$ implies $g_{i+1} \neq 1$ in $G$ (subscripts modulo $n$ ). The equation $s(t)=1$ is said to have a solution over $G$, or is solvable, if there is an embedding $\phi$ of $G$ into a group $H$ and an element $h \in H$ such that $\phi\left(g_{1}\right) h^{\varepsilon_{1}} \phi\left(g_{2}\right) h^{\varepsilon_{2}} \ldots \phi\left(g_{n}\right) h^{\varepsilon_{n}}=1$ in $H$. It is readily seen that $s(t)=1$ has a solution over $G$ if and only if the natural map $g \rightarrow g$ from $G$ to $\langle G, t \mid s(t)\rangle$ is injective. A conjecture stated in [15] by Levin asserts that if $G$ is torsion-free then every equation over $G$ is solvable. Prishchepov [16], using in particular results of Brodskii and Howie [2], has shown that the conjecture is true for equations of length at most six. A different proof of the same theorem was given by Ivanov and Klyachko [12]. Here we prove the following.

Theorem Every equation of length seven is solvable over any torsion-free group.

Levin [15] proved that an equation in which the exponent sum $\varepsilon_{1}+\varepsilon_{2}+\ldots+\varepsilon_{n}$ equals the length $n$ is solvable over any group; Stallings [17] showed that if cyclically the $t$ exponent $\varepsilon_{i}$ changes sign exactly twice then $s(t)=1$ is solvable over any torsion-free group; and Klyachko [14], again for torsion-free groups, has shown that if the exponent sum is \pm 1 then $s(t)=1$ is solvable. Let $G$ be torsion-free and $s(t)$ have length seven. 
Working modulo cyclic permutation and inversion it can be assumed without any loss that there are at most three occurrences of $t^{-1}$ in $s(t)$. If there are no occurrences then $s(t)=1$ is solvable by [15]; if there is exactly one or there are exactly two consecutive occurrences then $s(t)=1$ is solvable by [17]; or if there are exactly three then $s(t)=1$ is solvable by [14]. It follows that there remains to be considered two equations of length seven: $s_{1}(t)=g_{1} t g_{2} t g_{3} t g_{4} t g_{5} t^{-1} g_{6} t g_{7} t^{-1}=1$; and $s_{2}(t)=g_{1} g_{2} t g_{3} t g_{4} t^{-1} g_{5} t g_{6} t g_{7} t^{-1}=1$. Klyachko's approach was developed further by Fenn and Rourke [8], [9]; by Clifford and Goldstein [4], [5], [6]; and, in its most general form to date, by Clark [3]. The equation $s_{1}(t)=1$ is solvable by the main theorem in [3] (indeed it is solvable by a result in [4]). However $s_{2}(t)=1$ satisfies conditions (a) and (b) of Clark's theorem but not the third condition (c). The remainder of this paper will be devoted to solving $s_{2}(t)=1$. After some preliminaries in Section 2, the theorem is proved in Section 3.

\section{Preliminaries}

All necessary definitions concerning relative presentations and pictures can be found in [1]. A relative (group) presentation is a presentation of the form $\mathcal{P}=\langle G, \boldsymbol{x} \mid \boldsymbol{r}\rangle$ where $\boldsymbol{r}$ is a set of cyclically reduced words in $G *\langle\boldsymbol{x}\rangle$. If the relative presentation $\mathcal{P}$ is orientable and aspherical then the natural map from $G$ to $\langle G, \boldsymbol{x} \mid \boldsymbol{r}\rangle$ is injective [1]. In our case $\boldsymbol{x}, \boldsymbol{r}$ consists of the single element $t, s_{2}(t)$ (respectively), therefore $\mathcal{P}$ is orientable and so asphericity implies $s_{2}(t)=1$ is solvable. We use two methods to establish asphericity: weight test [1] and curvature distribution [7].

The star graph $\Gamma$ of $\mathcal{P}=\langle G, \boldsymbol{x} \mid \boldsymbol{r}\rangle$ has vertex set $\boldsymbol{x} \cup \boldsymbol{x}^{-1}$ and edge set $\boldsymbol{r}^{*}$, where $\boldsymbol{r}^{*}$ is the set of all cyclic permutations of the elements of $\boldsymbol{r} \cup \boldsymbol{r}^{-1}$ which begin with an element of $\boldsymbol{x} \cup \boldsymbol{x}^{-1}$. For $R \in \boldsymbol{r}^{*}$ write $R=S g$ where $g \in G$ and $S$ begins and ends with $\boldsymbol{x}$ symbols. Then $\iota(R)$ is the inverse of the last symbol of $S, \tau(R)$ the first symbol of $S$ and $\lambda(R)=g$. A weight function $\theta$ on $\Gamma$ is a real-valued function on the set of edges of $\Gamma$ which satisfies $\theta(S h)=\theta\left(S^{-1} h^{-1}\right)$. A weight function $\theta$ is called aspherical if the following three conditions are satisfied:

(W1) Let $R \in \boldsymbol{r}^{*}$ with $R=x_{1}^{\varepsilon_{1}} g_{1} \ldots x_{n}^{\varepsilon_{n}} g_{n}$. Then

$$
\sum_{i=1}^{n}\left(1-\theta\left(x_{i}^{\varepsilon_{i}} g_{i} \ldots x_{n}^{\varepsilon_{n}} g_{n} x_{1}^{\varepsilon_{1}} g_{1} \ldots x_{i-1}^{\varepsilon_{i-1}} g_{i-1}\right)\right) \geq 2 .
$$

(W2) Each admissible cycle in $\Gamma$ has weight at least 2 (where admissible means having a label trivial in $G$ ).

(W3) Each edge of $\Gamma$ has a non-negative weight.

If $\Gamma$ admits an aspherical weight function then $\mathcal{P}$ is aspherical [1]. 


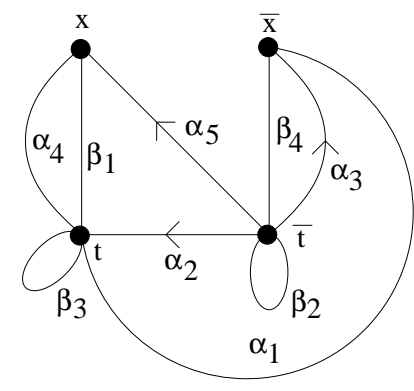

(i)

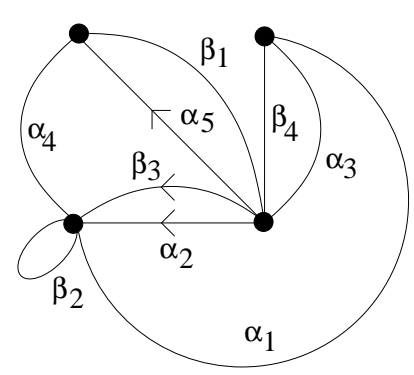

(ii)

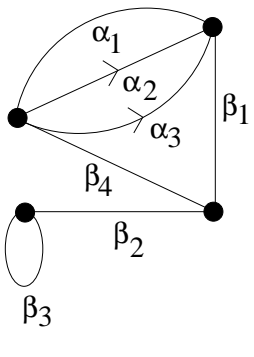

(iii)

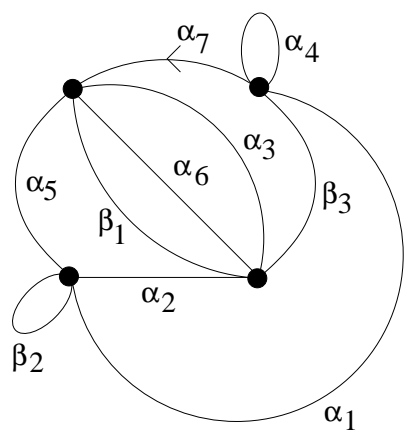

(iv)

Figure 2.1: star graphs

For convenience we rewrite $s_{2}(t)=1$ as

$$
s_{2}(t)=a t b t c t d t^{-1} \text { etftgt } t^{-1}=1
$$

where $a, d, e, g \in G \backslash\{1\}$ and $b, c, f \in G$. Moreover by applying the transformation $s=t b$, if necessary, it can be assumed without any loss that $b=1$ in $G$.

Lemma 2.1 The presentation $\mathcal{P}=\left\langle G, t \mid s_{2}(t)\right\rangle$ is aspherical if one of the following sets of conditions holds: (i) $a=e^{-1}$ and $d=g^{ \pm 1}$; (ii) $a=e^{-1}$ and $b=c=f$; (iii) $a \neq e^{-1}$, $b=f$ and $c=d=g$; (iv) $a=c=e^{-1}=f$; (v) $a=e^{-1}$ and $c=f=g^{-1}$; or (vi) $a=e^{-1}$, $b=c$ and $f=g^{-1}$.

Proof In each case a new generator $x$ will be introduced to obtain the presentation $\mathcal{Q}=$ $\left\langle G, t, x \mid r_{1}, r_{2}\right\rangle$. We show that there is an aspherical weight function $\theta$ for the star graph $\Gamma$ of $\mathcal{Q}$. It follows that $\mathcal{Q}$, and therefore $\mathcal{P}$, is aspherical.

(i) The relator $s_{2}(t)$ is given by $s_{2}(t)=a t^{2} c t d t^{-1} a^{-1} t f t d^{ \pm 1} t^{-1}$. Letting $x=t d^{ \pm 1} t^{-1} a t$ we obtain $r_{1}=x t c t d x^{-1} t d^{ \pm 1} f$ and $r_{2}=x^{-1} t d^{ \pm 1} t^{-1} a t$. The star graph $\Gamma$ for $\mathcal{Q}$ is given by Figure 2.1(i) in which (using $r_{1}$ ) $\alpha_{1}=1, \alpha_{2}=c, \alpha_{3}=d, \alpha_{4}=1$, $\alpha_{5}=d^{ \pm 1} f$; and (using $\left.r_{2}\right) \beta_{1}=1, \beta_{2}=d^{ \pm 1}, \beta_{3}=a, \beta_{4}=1$. Assign the weights $\theta\left(\alpha_{1}\right)=\theta\left(\alpha_{5}\right)=\theta\left(\beta_{2}\right)=\theta\left(\beta_{3}\right)=0$ and weight 1 to each of the remaining edges of $\Gamma$. Then $\Sigma\left(1-\theta\left(\alpha_{i}\right)\right)=\Sigma\left(1-\theta\left(\beta_{j}\right)\right)=2$ shows that $\mathbf{W} \mathbf{1}$ is satisfied; each cycle in $\Gamma$ of weight less than 2 has label $a^{m}$ or $d^{m}(m \neq 0)$ and so $G$ torsion-free implies W2 is satisfied; and W3 clearly holds.

(ii) $s_{2}(t)=a t^{3} d t^{-1} a^{-1} t^{2} g t^{-1} ; r_{1}=x t d t x^{-1} t g ; r_{2}=x^{-1} t^{-1} a t^{2} ; \Gamma$ is given by Figure 2.1(ii) in which $\alpha_{1}=1, \alpha_{2}=d, \alpha_{3}=\alpha_{4}=1, \alpha_{5}=g$ and $\beta_{1}=1, \beta_{2}=a, \beta_{3}=\beta_{4}=1$; and $\theta$ is defined by assigning 0 to edges $\alpha_{1}, \alpha_{5}, \beta_{1}, \beta_{2}$ and 1 to the remaining edges of $\Gamma$.

(iii) $s_{2}(t)=a t^{2} c t c t^{-1} e t^{2} c t^{-1} ; r_{1}=x^{2} e x a ; r_{2}=x^{-1} t^{2} c t^{-1} ; \Gamma$ is given by Figure 2.1(iii) in which $\alpha_{1}=1, \alpha_{2}=e, \alpha_{3}=a$ and $\beta_{1}=\beta_{2}=1, \beta_{3}=c, \beta_{4}=1$; and $\theta$ is defined by 
$\theta\left(\alpha_{1}\right)=\theta\left(\beta_{2}\right)=\theta\left(\beta_{3}\right)=0, \theta\left(\beta_{1}\right)=\theta\left(\beta_{4}\right)=1$ and either $\theta\left(\alpha_{2}\right)=1, \theta\left(\alpha_{3}\right)=0$ when $a^{ \pm 1}=e^{2}$ or $\theta\left(\alpha_{2}\right)=0, \theta\left(\alpha_{3}\right)=1$ when $a^{2}=e^{ \pm 1}$ or $\theta\left(\alpha_{2}\right)=\theta\left(\alpha_{3}\right)=\frac{1}{2}$ otherwise.

(iv) $s_{2}(t)=c t^{2} c t d t^{-1} c^{-1} t c t g t^{-1} ; r_{1}=x t^{2} x d x^{-1} t x g ; r_{2}=x^{-1} t^{-1} c t$; and $\Gamma$ is given by Figure 2.1(iv) in which $\alpha_{1}=\alpha_{2}=\alpha_{3}=1, \alpha_{4}=d, \alpha_{5}=\alpha_{6}=1, \alpha_{7}=g$ and $\beta_{1}=1$, $\beta_{2}=c, \beta_{3}=1$; and $\theta$ is defined by assigning 0 to the edges $\alpha_{4}, \alpha_{5}, \beta_{2}, \beta_{3}$ and 1 to the remaining edges of $\Gamma$.

(v) $s_{2}(t)=a t^{2} c t d t^{-1} a^{-1} t c t c^{-1} t ; r_{1}=x^{-1} t c t d x t ; r_{2}=x^{-1} t^{-1} a^{-1} t c t$; and $\Gamma$ is given by Figure 2.1(ii) when we take $r_{1}=x^{-1} \alpha_{4} t \alpha_{2} t \alpha_{5} x \alpha_{1} t \alpha_{3}$ so that $\alpha_{1}=\alpha_{3}=\alpha_{4}=1$, $\alpha_{2}=c, \alpha_{5}=d$ and $\beta_{1}=1, \beta_{2}=a^{-1}, \beta_{3}=c, \beta_{4}=1$; and $\theta$ is defined as in (ii).

(vi) $s_{2}(t)=a t^{3} d t^{-1} a^{-1} t f t f^{-1} t^{-1} ; r_{1}=x^{-1} t^{2} d x t ; r_{2}=x^{-1} t^{-1} a^{-1} t f t$; $\Gamma$ is given by Figure 2.1(ii) again when $r_{1}=x^{-1} \alpha_{4} t \alpha_{2} t \alpha_{5} x d_{1} t \alpha_{3}$ and so $\alpha_{1}=\alpha_{2}=\alpha_{3}=\alpha_{4}=1, \alpha_{5}=d$ and $\beta_{1}=1, \beta_{2}=a^{-1}, \beta_{3}=f, \beta_{4}=1$; and once more $\theta$ of (ii) suffices.

Lemma 2.2 The equation $s_{2}(t)=1$ is solvable if one of the following sets of conditions holds: (i) $a=e$ and $d=g$; (ii) $a=e$ and $b=f$; (iii) $c=f$ and $d=g$; (iv) $a=e=f^{-1}$ and $b=c$.

Proof If (i) holds then adding the generator $x=t d t^{-1}$ at yields $\mathcal{P}=\langle G, x \mid r(x)\rangle$ where $r(x)=x f x^{2} c x f x a^{-1} x^{-1} f^{-1} x^{-1} c^{-1} d^{-1} c$; if (ii) holds then $x=t^{-1} a t^{2}$ and $r(x)=x d x g x c d x g x c a^{-1} c^{-1} x^{-1} g^{-1} x^{-1} d^{-1}$; if (iii) holds then $x=t c t d t^{-1}$ and $r(x)=\operatorname{exac}^{-1} x \operatorname{exaxa}^{-1} x^{-1} e^{-1} x^{-1} d^{-1}$; and if (iv) holds then $x=t^{-1} a t a^{-1} t$ and $r(x)=x g x^{3} d x g x^{-1} g^{-1} d^{-1} a d$. In each case the equation is solvable [17]. For example if $a=e$ and $d=g$ then $\mathcal{P}=\left\langle G, t \mid a t^{2} c t d t^{-1} a t f t d t^{-1}\right\rangle=\langle G, t, x| a t^{2} c t d t^{-1}$ etftdt $\left.t^{-1}, x^{-1} t d t^{-1} a t\right\rangle=$ $\left\langle G, t, x \mid t c x f x, x^{-1} t d t^{-1} a t\right\rangle=\left\langle G, x \mid x f x^{2} c x f x a^{-1} x^{-1} f^{-1} x^{-1} c^{-1} d^{-1} c\right\rangle$.

\section{Proof of theorem}

Recall that $\mathcal{P}=\left\langle G, t \mid s_{2}(t)\right\rangle$ where $s_{2}(t)=$ atbtctdt $^{-1}$ etftgt $t^{-1}(a, d, e, g \in G \backslash\{1\} ; b=1$; $c, f \in G$ ). If $G$ is locally indicable [10] then $s_{2}(t)=1$ is solvable, so we shall assume otherwise: in particular, $G$ is not cyclic. Furthermore it can be assumed without any loss that $G=\langle a, b, c, d, e, f, g\rangle$. Given this, we show that either $\mathcal{P}$ is aspherical using, if necessary, Lemma 2.1 or that one of the conditions of Lemma 2.2 must hold and this suffices to prove the theorem.

Our approach will be to use the dual of pictures that were introduced by Howie, further details of which can be found in [11]. Suppose by way of contradiction that $K$ is a reduced spherical diagram over $\mathcal{P}$. The regions of $K$ are given (up to cyclic permutation and inversion) by Figure 3.1(i). The star graph $\Gamma$ of $\mathcal{P}$ is given by Figure 3.1(ii). 


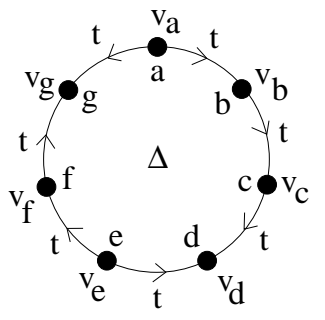

(i)

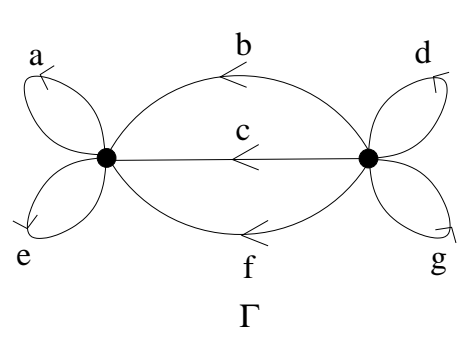

(ii)

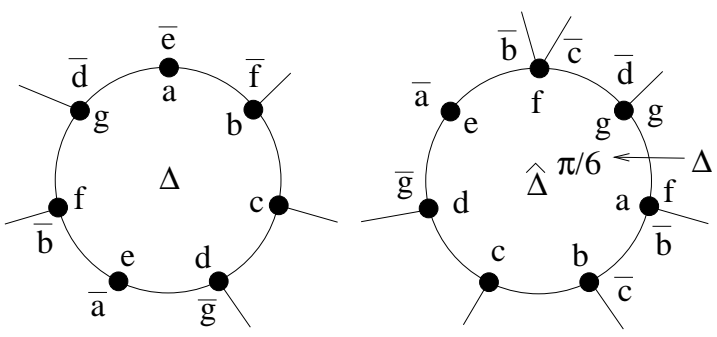

(iii)

(iv)

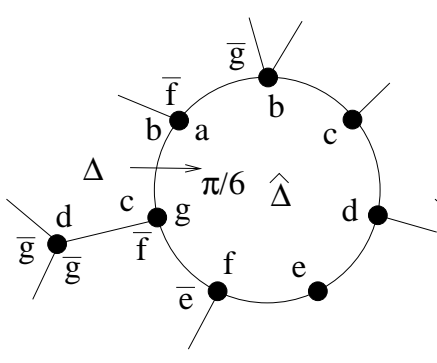

(v)

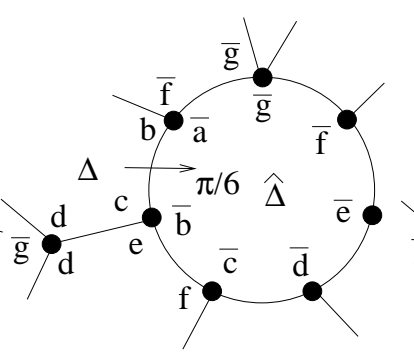

(vi)

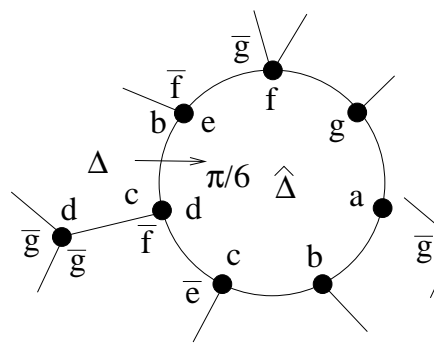

(vii)

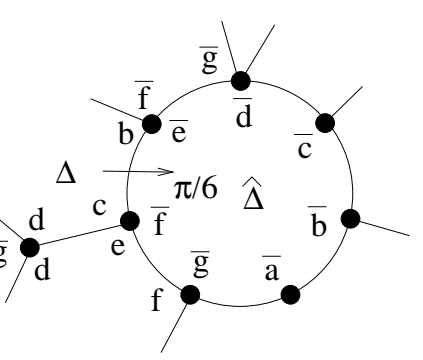

(viii)

Figure 3.1: region $\Delta$ of $K$, star graph $\Gamma$ of $P$; and regions $\Delta$ and $\hat{\Delta}$ for Case 1

The diagram $K$ will have a distinguished vertex $v_{0}$ (corresponding to the region of the spherical picture dual to $K)$ and, in general, $l\left(v_{0}\right)$ yields a cycle in $\Gamma$ which is not necessarily admissible, that is, $l\left(v_{0}\right)$ need not equal 1 in $G$.

Assume until otherwise stated (near the end of Section 3) that $l\left(v_{0}\right)=1$ in $G$. Then the product of the corner labels read anti-clockwise around any vertex of $K$ yields an admissible cycle in $\Gamma$. The degree of a vertex $v$ is denoted by $d(v)$. Define an angle function on $K$ by assigning $\frac{2 \pi}{d(v)}$ to each corner at a vertex $v$. This way the curvature of a vertex equals 0 . If $\Delta$ is a region of $K$ of degree $n$ with vertices $v_{1}, \ldots, v_{n}$ such that $d\left(v_{i}\right)=d_{i}(1 \leq i \leq n)$ then the curvature of $\Delta$ is $c(\Delta)=c\left(d_{1}, \ldots, d_{n}\right)=(2-n) \pi+\sum_{i=1}^{n} \frac{2 \pi}{d_{i}}$. It is a consequence of Euler's formula that $c(K):=\sum_{\Delta \in K} c(\Delta)=4 \pi$, in particular, $K$ must contain regions

of positive curvature $(*)$. Using $\Gamma$ (and the fact that $G$ is torsion-free) we see that the possible labels of vertices of degree 2 in $K$ are (up to cyclic permutation and inversion)

$$
S=\left\{a e^{ \pm 1}, d g^{ \pm 1}, b c^{-1}, b f^{-1}, c f^{-1}\right\}
$$

We will proceed according to the number $N$ of labels in $S$ that are admissible. The possibilities can be reduced as follows. We can work modulo equivalence, that is, modulo inversion, cyclic permutation, $t \leftrightarrow t^{-1}$ and $(a, b, c, d, e, f, g) \leftrightarrow\left(d^{-1}, c^{-1}, b^{-1}, a^{-1}, g^{-1}, f^{-1}, e^{-1}\right)$. For example $a e, d g^{-1}$ is equivalent to $a e^{-1}, d g$. Lemmas 2.1(i)-(ii) and 2.2(i)-(iii) can be applied to reduce the number of cases: for example at most one of $a e^{ \pm 1}, d g^{ \pm 1}$ is admissible. Given this, it quickly follows that $N \leq 3$. Observe that if $N=0$ then 


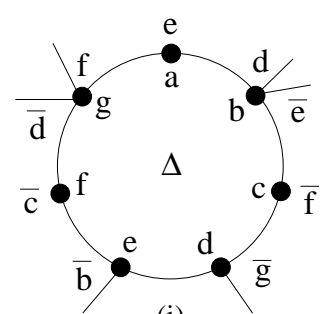

(i)

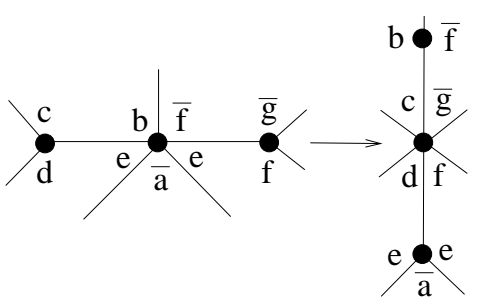

(v)

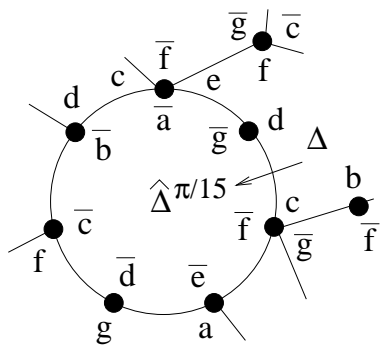

(ix)
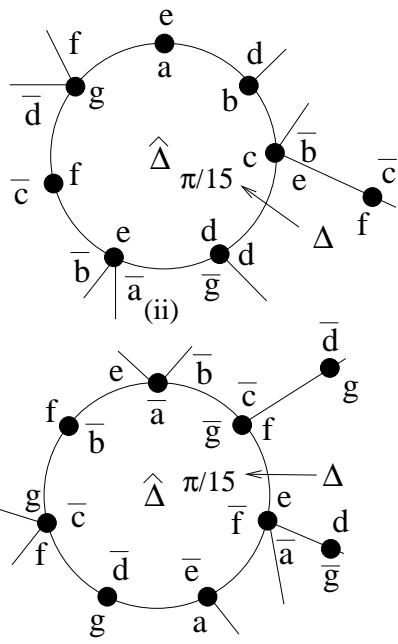

(vi)

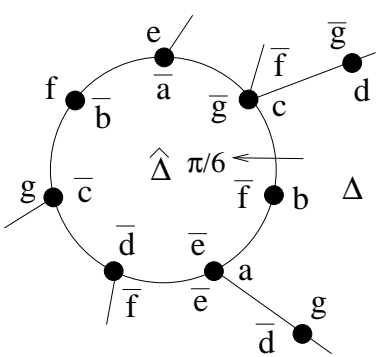

(x)
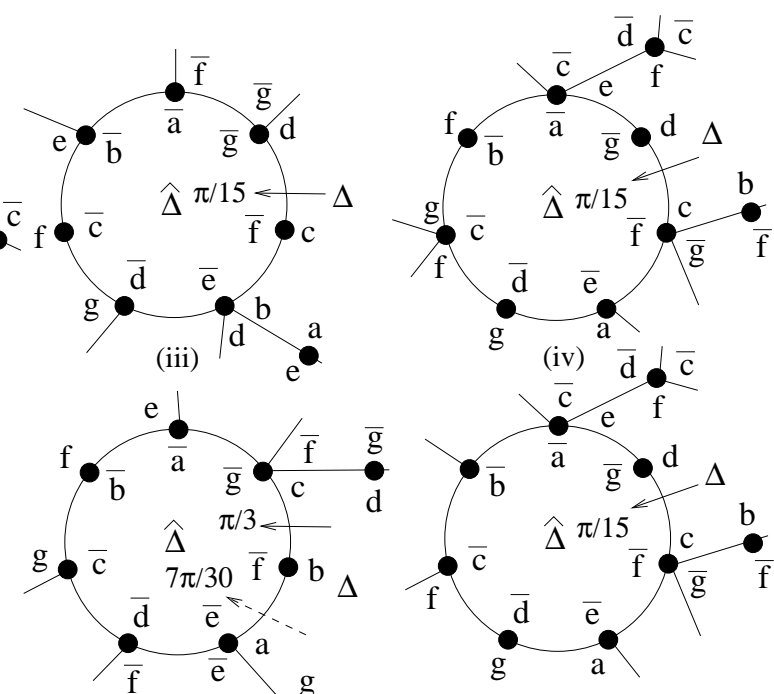

(vii) $\overline{\mathrm{d}} \mathrm{g}^{\mathrm{g}}$

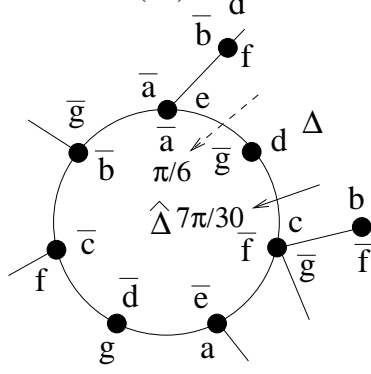

(xi)

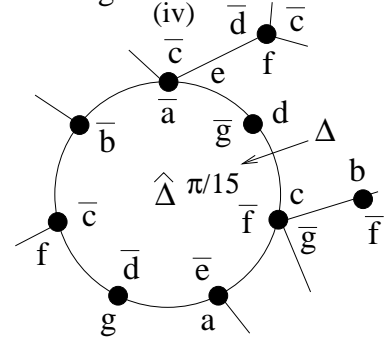

(viii)

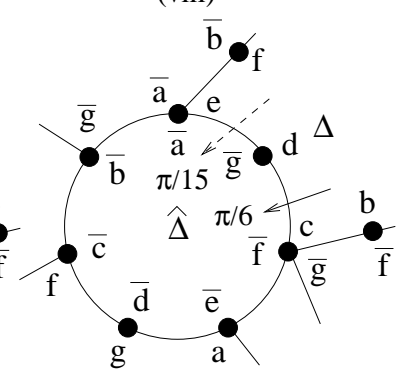

(xii)

Figure 3.2: regions $\Delta$ and $\hat{\Delta}$ for Cases 5 and 8

$c(\Delta) \leq c(3,3,3,3,3,3,3)=-\frac{\pi}{3}$ for each region $\Delta$ of $K$, contradicting $(*)$, so it can be assumed that $N>0$. Checking shows that there are ten cases that remain to be considered: $(N=1)$ Case 1: $a=e$; Case 2: $a=e^{-1}$; Case 3: $b=f$; Case 4: $b=c ;(N=2)$ Case 5: $a=e^{-1}, c=f$; Case 6: $a=e^{-1}, b=f$; Case 7: $a=e^{-1}, b=c$; Case 8: $b=f, d=g$; Case 9: $a=e, b=c$; and $(N=3)$ Case 10: $b=c=f$.

Remark 1 In what follows much use will be made, often without explicit mention, of the fact that vertex labels correspond to closed paths in the star graph $\Gamma$ and of the assumptions that $G$ is torsion-free, $G$ is non-cyclic and that $a, d, e, g$ are non-trivial elements in $G$. Indeed, unless otherwise stated, contradiction will mean a contradiction to one of these assumptions.

Remark 2 Since $c(2,3,3,3,3,3,3)=c(2,2,3,3,3,4,4)=0$ it follows that if $c(\Delta)>0$ then $\Delta$ has at least two vertices of degree 2 ; and if $\Delta$ has exactly two vertices of degree 2 then at most one of the remaining vertices has degree $>3$.

Notation Suppose that $l(v) \in\left\{w w_{1}, w w_{2}, \ldots, w w_{k}\right\}$. In what follows we use the notation 
$l(v)=w\left\{w_{1}, w_{2}, \ldots, w_{k}\right\}$ to indicate this. For example, $l\left(v_{g}\right)=g f\left\{b^{-1}, c^{-1}\right\}$ will mean $l\left(v_{g}\right) \in\left\{g f b^{-1}, g f c^{-1}\right\}$.

From now on let $\Delta$ be a region of $K$ such that $c(\Delta)>0$. In Cases 1, 5, 8 and 9 we will show that $\Delta$ has a neighbouring region $\hat{\Delta}$, uniquely associated with $\Delta$, such that $c(\hat{\Delta})+c(\Delta) \leq 0$. It follows that the total curvature $c(K)$ of $K$ is non-positive, which contradicts $c(K)=4 \pi$.

Case 1: $a=e$. Since $c(\Delta)>0, l\left(v_{a}\right)=a e^{-1}$ and $l\left(v_{e}\right)=e a^{-1}$ as shown in Figure 3.1(iii). First suppose that each of the remaining vertices of $\Delta$ has degree 3. Then $l\left(v_{g}\right) \in$ $\left\{g^{2} d^{-1}, g d^{-2}\right\}$. But if $l\left(v_{g}\right)=g d^{-2}$ then $l\left(v_{f}\right)=b^{-1} f c^{-1} w$ and so (see the star graph ए) $d(f)>3$, so let $l\left(v_{g}\right)=g^{2} d^{-1}$ forcing $l\left(v_{f}\right)=b^{-1} f a$ and $l\left(v_{d}\right)=d g^{-2}$. Given this, $l\left(v_{b}\right)=f^{-1} b\left\{a^{ \pm 1}, e^{ \pm 1}\right\}$. But $l\left(v_{b}\right)=f^{-1} b\{a, e\}$ implies $a^{2}=1$, and if $l\left(v_{b}\right)=f^{-1} b\left\{a^{-1}, e^{-1}\right\}$ then $d\left(v_{c}\right)>3$. Now suppose that exactly one of the remaining vertices of $\Delta$ has degree $>3$. If $d\left(v_{b}\right)>3$ or $d\left(v_{c}\right)>3$ or $d\left(v_{d}\right)>3$ then $l\left(v_{g}\right)=g^{2} d^{-1}$ and $l\left(v_{f}\right)=b^{-1} f a$ as shown in Figure 3.1(iv); and if $d\left(v_{f}\right)>3$ or $d\left(v_{g}\right)>3$ then $l\left(v_{b}\right)=f^{-1} b\left\{a^{ \pm 1}, e^{ \pm 1}\right\}$ and each choice uniquely determines $l\left(v_{c}\right)$ and $l\left(v_{d}\right)$ (otherwise there is a contradiction) as shown in Figure 3.1(v)-(viii). In all cases add $c(\Delta) \leq c(2,2,3,3,3,3,4)=\frac{\pi}{6}$ to $c(\hat{\Delta})$ as shown in Figure 3.1(iv)-(viii). Observe that in Figure 3.1(iv) if $d\left(v_{e}\right)>2$ in $\hat{\Delta}$ then $c(\hat{\Delta}) \leq-\frac{\pi}{3}$, or if $d\left(v_{e}\right)=2$ (as shown) then $d\left(v_{f}\right)>3$ in $\hat{\Delta}$ and $c(\hat{\Delta}) \leq c(2,3,3,3,3,3,4)=-\frac{\pi}{6}$. Observe also that $d\left(v_{b}\right)>3, d\left(v_{g}\right)>3, d\left(v_{f}\right)>3, d\left(v_{d}\right)>3$ in $\hat{\Delta}$ of Figure 3.1(v)-(viii) respectively (otherwise there is a contradiction) and again $c(\hat{\Delta}) \leq-\frac{\pi}{6}$ in each case. Finally, checking Figure 3.1(iv)-(viii) shows that $\hat{\Delta}$ does not receive positive curvature across more than one edge; for example, if $\hat{\Delta}$ of Figure 3.1(iv) coincides with $\hat{\Delta}$ of Figure 3.1(vii) then $a=e=f^{-1}$ from (iv) and $a=e=f$ from (vii), a contradiction. It follows that $\hat{\Delta}$ is uniquely associated with $\Delta$ in each case and $c(\hat{\Delta})+c(\Delta) \leq-\frac{\pi}{6}+\frac{\pi}{6}=0$.

Case 5: $a=e^{-1}$ and $c=f$. If $l\left(v_{a}\right)=a e$ and $d\left(v_{g}\right)=3$ then $l\left(v_{g}\right)=g f\left\{b^{-1}, c^{-1}\right\}$ which implies $g=1$ or $g=f^{-1}$ and the presentation $\mathcal{P}$ is aspherical by Lemma 2.1(v); if $l\left(v_{c}\right)=c f^{-1}$ and $d\left(v_{b}\right)=3$ then $l\left(v_{b}\right)=b e^{-1}\left\{c^{-1}, f^{-1}\right\}$ and Lemma 2.1(iv) applies; if $l\left(v_{e}\right)=e a$ and $d\left(v_{f}\right)=3$ then $l\left(v_{f}\right)=g f\left\{b^{-1}, c^{-1}\right\}$ and again either $g=1$ or Lemma 2.1(v) applies; if $l\left(v_{f}\right)=f c^{-1}$ and $d\left(v_{e}\right)=3$ then $l\left(v_{e}\right)=e b^{-1}\{c, f\}$ and Lemma 2.1(iv) applies; or if $l\left(v_{c}\right)=c f^{-1}$ and $l\left(v_{e}\right)=e a$ then $l\left(v_{d}\right)=g^{-1} d b w$ and $d\left(v_{d}\right)>3$. Since $c(\Delta)>0$, it follows that we must have $d\left(v_{a}\right)=d\left(v_{c}\right)=d\left(v_{f}\right)=2$ as shown in Figure 3.2(i). This implies that each of $d\left(v_{b}\right), d\left(v_{e}\right)$ and $d\left(v_{g}\right)$ is greater than 3 and so $l\left(v_{d}\right) \in\left\{d^{2} g^{-1}, d g^{-2}\right\}$. If $l\left(v_{d}\right)=d^{2} g^{-1}$ then $d\left(v_{b}\right)=d\left(v_{g}\right)=4$ or $d\left(v_{e}\right)=d\left(v_{g}\right)=4$ yields a contradiction and so $d\left(v_{b}\right)=d\left(v_{e}\right)=4, d\left(v_{g}\right)=5$ and add $c(\Delta) \leq c(2,2,2,3,4,4,5)=\frac{\pi}{15}$ to $c(\hat{\Delta})$ as shown in Figure 3.2(ii). Observe (as noted above) that $d\left(v_{c}\right)>3$ in $\hat{\Delta}$ of Figure 3.2(ii) and moreover $l\left(v_{e}\right)=a^{-1}$ ew forces $d\left(v_{e}\right)>3$, so if $d\left(v_{a}\right)>2$ or $d\left(v_{f}\right)>2$ in $\hat{\Delta}$ then $c(\hat{\Delta}) \leq-\frac{\pi}{3}$; on the other hand if $d\left(v_{a}\right)=d\left(v_{f}\right)=2$ as shown then $d\left(v_{g}\right)>3$ and $c(\hat{\Delta}) \leq-\frac{\pi}{6}$. Now suppose that $l\left(v_{d}\right)=d g^{-2}$. Again $d\left(v_{b}\right)=d\left(v_{g}\right)=4$ or $d\left(v_{e}\right)=d\left(v_{g}\right)=4$ does not occur and so add $c(\Delta) \leq \frac{\pi}{15}$ to $c(\hat{\Delta})$ as shown in Figure 3.2(iii). Observe that $d\left(v_{e^{-1}}\right)>3$ in $\hat{\Delta}$, and, moreover, $d\left(v_{a^{-1}}\right)>3$ otherwise $l\left(v_{a}^{-1}\right)=a^{-1} f^{-1} c$ and the fact that $l\left(v_{e}\right)=a^{-1} e b^{-1} w$ in $\Delta$ now forces $d\left(v_{e}\right)>4$ and $c(\Delta)<0$. If $d\left(v_{c^{-1}}\right)>2$ in $\hat{\Delta}$ then $c(\hat{\Delta}) \leq-\frac{\pi}{3}$ so let $d\left(v_{c^{-1}}\right)=2$ 
as shown. But now if $d\left(v_{b^{-1}}\right)=3$ in $\hat{\Delta}$ then Lemma 2.1(iv) applies, so assume otherwise. It follows that $c(\hat{\Delta})+c(\Delta) \leq-\frac{\pi}{6}+\frac{\pi}{15}<0$ and checking Figure 3.2(ii), (iii) shows that $\hat{\Delta}$ is uniquely associated with $\Delta$.

Case 8: $b=f$ and $d=g$. There are seven subcases: (i) $d\left(v_{b}\right)=d\left(v_{d}\right)=2 ;$ (ii) $d\left(v_{b}\right)=$ $d\left(v_{f}\right)=2$; (iii) $d\left(v_{b}\right)=d\left(v_{g}\right)=2$; (iv) $d\left(v_{d}\right)=d\left(v_{f}\right)=2 ;(\mathrm{v}) d\left(v_{d}\right)=d\left(v_{g}\right)=2$; (vi) $d\left(v_{b}\right)=d\left(v_{d}\right)=d\left(v_{g}\right)=2 ;(\operatorname{vii}) d\left(v_{b}\right)=d\left(v_{d}\right)=d\left(v_{f}\right)=2$. In subcase (i) if $d\left(v_{c}\right)=3$ then $g=c=d$ and $\mathcal{P}$ is aspherical by Lemma 2.1(iii);or if $d\left(v_{a}\right)=d\left(v_{g}\right)=3$ then $l\left(v_{a}\right)=a^{2} e^{-1}$ and $l\left(v_{g}\right)=g b c^{-1}$ and again Lemma 2.1(iii) applies. In subcase (ii) if $d\left(v_{c}\right)=3$ or if $d\left(v_{g}\right)=3$ then $g=c=d$ and Lemma 2.1(iii) applies. In subcase (iii) $d\left(v_{c}\right) \geq 4$ so $c(\Delta)>0$ forces $l\left(v_{a}\right)=a e^{-2}$ and either $l\left(v_{f}\right)=f c^{-1} d^{-1}$ and $l\left(v_{e}\right)=e c^{-1}\{b, f\}$ or $l\left(v_{f}\right)=f c^{-1} g^{-1}$ and $l\left(v_{e}\right)=e f^{-1} c$. But in each case $G$ is then cyclic. In subcase (iv) $d\left(v_{g}\right) \geq 4$ so $c(\Delta)>0$ forces $l\left(v_{e}\right)=e a^{-2}$ and either $l\left(v_{c}\right)=c f^{-1} d$ and $l\left(v_{b}\right)=b e c^{-1}$ or $l\left(v_{c}\right)=c f^{-1} g$ and $l\left(v_{b}\right)=b a c^{-1}$ and again $G$ is then cyclic. In subcase (v) $d\left(v_{e}\right)=d\left(v_{f}\right)=3$ forces $l\left(v_{e}\right)=a^{-1} e^{2}$ and $l\left(v_{f}\right)=d f c^{-1}$ so $g=c=d$ and Lemma 2.1(iii) applies;or if $d\left(v_{a}\right)=d\left(v_{b}\right)=3$ then $l\left(v_{a}\right)=e^{-1} a^{2}$ and $l\left(v_{b}\right)=g b c^{-1}$ and once more Lemma 2.1(iii) applies. It follows that in subcases (i)-(v) $c(\Delta)>0$ yields a contradiction or $\mathcal{P}$ is aspherical by Lemma 2.1(iii). This leaves (vi) and (vii) to be considered. First consider subcase (vi). The fact that $l\left(v_{b}\right)=b f^{-1}$ and $l\left(v_{d}\right)=d g^{-1}$ in $\Delta$ means that if $d\left(v_{c}\right)=3$ then $l\left(v_{c}\right)=g^{-1} c f^{-1}$ which implies $g=c$ and Lemma 2.1(iii) applies so assume that $d\left(v_{c}\right)>3$. If $d\left(v_{e}\right)=d\left(v_{f}\right)=3$ then $l\left(v_{e}\right) \in\left\{e a^{-2}, e^{2} a^{-1}\right\}$. But $l\left(v_{e}\right)=e a^{-2}$ forces $d\left(v_{f}\right)>3$ and $l\left(v_{e}\right)=e^{2} a^{-1}$ forces $l\left(v_{f}\right)=d f c^{-1}$ and Lemma 2.1(iii) applies, so assume otherwise.

Let $d\left(v_{a}\right)=d\left(v_{f}\right)=3$ in $\Delta$. Then $l\left(v_{a}\right)=a e^{-2}$ and $l\left(v_{f}\right)=f c^{-1}\left\{d^{-1}, g^{-1}\right\}$. First let $l\left(v_{f}\right)=f c^{-1} d^{-1}$. Then $d\left(v_{c}\right)>4$ and $d\left(v_{e}\right)>4$ otherwise there is a contradiction. Moreover if $l\left(v_{e}\right)=5$ then $l\left(v_{e}\right)=a^{-1} e c^{-1}\left\{d^{ \pm 1} b, d^{ \pm 1} c, d^{ \pm 1} f, g^{ \pm 1} b, g^{ \pm 1} c, g^{ \pm 1} f, b a^{-1}, f a^{-1}, b e^{ \pm 1}, f e^{ \pm 1}\right\}$ and in each case $A$ is cyclic, a contradiction. Add $c(\Delta) \leq c(2,2,2,3,3,5,6)=\frac{\pi}{15}$ to $c(\hat{\Delta})$ as shown in Figure 3.2(iv). Observe that if $d\left(v_{b^{-1}}\right)>2$ or $d\left(v_{d^{-1}}\right)>2$ in $\hat{\Delta}$ then $c(\hat{\Delta}) \leq c(3,3,3,5,6)=-\frac{4 \pi}{15}$; or if $d\left(v_{b^{-1}}\right)=d\left(v_{d^{-1}}\right)=2$ as shown then $d\left(v_{c^{-1}}\right)>3$ and $c(\hat{\Delta}) \leq c(3,4,5,6)=-\frac{\pi}{10}$. Now let $l\left(v_{f}\right)=f c^{-1} g^{-1}$. Then again $d\left(v_{c}\right)>4$ and $d\left(v_{e}\right)>4$ otherwise there is a contradiction. Moreover if $d\left(v_{e}\right)=5$ then there is a contradiction except when $l\left(v_{e}\right)=a^{-1} e f^{-1} b e$. But if we assume that, subject to having a minimum number of regions, $K$ has a maximum number of vertices of degree 2 , then $l\left(v_{e}\right)=a^{-1} e f^{-1} b e$ does not occur since the bridge move [1] shown in Figure 3.2(v) increases the number of vertices of degree 2 (and does not change the number of regions) in $K$. Add $c(\Delta) \leq \frac{\pi}{15}$ to $c(\hat{\Delta})$ as shown in Figure 3.2(vi). If $d\left(v_{b^{-1}}\right)>2$ or $d\left(v_{d^{-1}}\right)>2$ in $\hat{\Delta}$ then $c(\hat{\Delta}) \leq-\frac{\pi}{3}$; or if $d\left(v_{b^{-1}}\right)=d\left(v_{d^{-1}}\right)=2$ as shown then $d\left(v_{a^{-1}}\right)>3$ and $d\left(v_{c^{-1}}\right)>3$ and again $c(\hat{\Delta}) \leq-\frac{\pi}{3}$.

Let $d\left(v_{a}\right)=d\left(v_{e}\right)=3$ in $\Delta$. Then $l\left(v_{a}\right)=a e^{-2}$ and so $l\left(v_{e}\right)=a^{-1} e^{2}$. Add $c(\Delta) \leq \frac{\pi}{3}$ to $c(\hat{\Delta})$ as shown in Figure 3.2(vii). Observe that $d\left(v_{d^{-1}}\right)>3$ in $\hat{\Delta}$ otherwise Lemma 2.1(iii) applies. If $d\left(v_{b^{-1}}\right)>2$ then $c(\hat{\Delta}) \leq-\frac{\pi}{3}$ by Lemmma 2.1(iii). If $d\left(v_{b^{-1}}\right)=2$ as shown then $d\left(v_{c^{-1}}\right)>3$. If now $d\left(v_{a^{-1}}\right)>3$ then $c(\hat{\Delta}) \leq-\frac{\pi}{3}$; or if $d\left(v_{a^{-1}}\right)=3$ then $l\left(v_{a^{-1}}\right)=a^{-1} e^{2}$ which forces $d\left(v_{c}\right)>4$ in $\Delta$ otherwise Lemma 2.1(iii) applies and in this 
case add $c(\Delta) \leq c(2,2,2,3,3,4,5)=\frac{7 \pi}{30}$ to $c(\hat{\Delta}) \leq c(2,2,3,3,4,4,5)=-\frac{4 \pi}{15}$ as shown by the dotted arrow.

Let $d\left(v_{e}\right)=3$ only. First let $l\left(v_{e}\right)=e a^{-2}$. Then $l\left(v_{a}\right)=e^{-1} a e^{-1} w$ and $l\left(v_{f}\right)=b^{-1} f c^{-1} w$ forces $d\left(v_{a}\right)>4, d\left(v_{f}\right)>4$ and $c(\Delta) \leq 0$. Now let $l\left(v_{e}\right)=e^{2} a^{-1}$. Then $c(\Delta)>0$ forces $l\left(v_{a}\right)=e^{-1} a e^{-1}\left\{b^{-1} f, f^{-1} b\right\}$. But a bridge move at $v_{a}$ similar to the one shown in Figure $3.2(\mathrm{v})$ yields a contradiction to the number of vertices of degree 2 being maximal. Therefore $c(\Delta) \leq 0$.

Let $d\left(v_{f}\right)=3$ only. If $l\left(v_{f}\right)=f c^{-1} d^{-1}$ then $d\left(v_{c}\right)>4$ and so $l\left(v_{e}\right)=a^{-1} e c^{-1}\{b, f\}$. Add $c(\Delta) \leq \frac{\pi}{15}$ to $c(\hat{\Delta})$ as shown in Figure $3.2\left(\right.$ viii) in which $l\left(v_{e}\right)$ in $\Delta$ forces $d\left(v_{b^{-1}}\right)>2$ in $\hat{\Delta}$ and so $c(\hat{\Delta}) \leq-\frac{\pi}{10}$. If $l\left(v_{f}\right)=f c^{-1} g^{-1}$ then again $d\left(v_{c}\right)>4$ and if $l\left(v_{e}\right)=a^{-1} e f^{-1} b$ then $a=e$, a contradiction, so $l\left(v_{e}\right)=a^{-1} e f^{-1} c$. Add $c(\Delta) \leq \frac{\pi}{15}$ to $c(\hat{\Delta})$ as shown in Figure $3.2($ ix $)$. Observe that $d\left(v_{b^{-1}}\right)>2$ in $\hat{\Delta}$ so $c(\hat{\Delta}) \leq-\frac{\pi}{10}$.

Finally for this subcase let $d\left(v_{a}\right)=3$ only. Add $c(\Delta) \leq \frac{\pi}{6}$ to $c(\hat{\Delta})$ as shown in Figure $3.2(\mathrm{x})$. If $d\left(v_{d^{-1}}\right)=3$ in $\hat{\Delta}$ then $l\left(v_{d^{-1}}\right)=f^{-1} d^{-1}\{b, c\}$ which implies $d=1$ or $d=c$ and Lemma 2.1(iii) applies, so assume that $d\left(v_{d^{-1}}\right)>3$. If now $d\left(v_{b^{-1}}\right)>2$ then $c(\hat{\Delta}) \leq-\frac{\pi}{3}$; or if $d\left(v_{b^{-1}}\right)=2$ as shown then $d\left(v_{c^{-1}}\right)>3$ and $c(\hat{\Delta}) \leq-\frac{\pi}{6}$.

Now consider subcase (vii) and so $l\left(v_{b}\right)=b f^{-1}, l\left(v_{d}\right)=d g^{-1}$ and $l\left(v_{f}\right)=f b^{-1}$. If $l\left(v_{c}\right)=$ $g^{-1} c f^{-1}$ or $l\left(v_{g}\right)=c^{-1} g\{b, f\}$ then $g=c$ and Lemma 2.1(iii) applies so assume that $d\left(v_{c}\right)>3$ and $d\left(v_{g}\right)>3$. Let $d\left(v_{a}\right)=d\left(v_{e}\right)=3$. Then $l\left(v_{a}\right)=a^{2} e^{-1}$ and $l\left(v_{e}\right)=e a^{-2}$. If $d\left(v_{c}\right)=d\left(v_{g}\right)=4$ there is a contradiction, so add $c(\Delta) \leq c(2,2,2,3,3,4,5)=\frac{7 \pi}{30}$ to $c(\hat{\Delta})$ as shown in Figure 3.2(xi). If $d\left(v_{b^{-1}}\right)=3$ in $\hat{\Delta}$ then $g=1$ or $g=c$ and Lemma 2.1(iii) applies so assume $d\left(v_{b^{-1}}\right)>3$. If $d\left(v_{d^{-1}}\right)>2$ then $c(\hat{\Delta}) \leq-\frac{\pi}{3}$; or if $d\left(v_{d^{-1}}\right)=2$ as shown, $d\left(v_{c^{-1}}\right)>3$ and $d\left(v_{e^{-1}}\right)>3$ then $c(\hat{\Delta}) \leq-\frac{\pi}{3}$; or if $d\left(v_{d^{-1}}\right)=2, d\left(v_{c^{-1}}\right)>3$ and $d\left(v_{e^{-1}}\right)=3$ then this forces $d\left(v_{f^{-1}}\right)>4$ and $c(\hat{\Delta}) \leq-\frac{4 \pi}{15}$; or if $d\left(v_{d^{-1}}\right)=2, d\left(v_{c^{-1}}\right)=3$ and $d\left(v_{e^{-1}}\right)>3$ then $l\left(v_{c^{-1}}\right)=f c^{-1}\left\{d^{-1}, g^{-1}\right\}$ forces $d\left(v_{f^{-1}}\right)>4$ and again $c(\hat{\Delta}) \leq-\frac{4 \pi}{15}$; or if $d\left(v_{d^{-1}}\right)=2$ and $d\left(v_{c^{-1}}\right)=d\left(v_{e^{-1}}\right)=3$ then $l\left(v_{f}^{-1}\right)=g^{-1} c f^{-1} b w$ forces $d\left(v_{f}^{-1}\right)>5$, so add $c(\Delta) \leq c(2,2,2,3,3,4,6)=\frac{\pi}{6}$ to $c(\hat{\Delta}) \leq c(2,2,3,3,3,4,6)=-\frac{\pi}{6}$ as shown by the dotted arrow in Figure 3.2(xi). Let $d\left(v_{a}\right)=3$ only. Then any attempt at labelling to obtain $c(\Delta)>0$ yields a contradiction. Finally for this subcase let $d\left(v_{e}\right)=3$ only. Add $c(\Delta) \leq \frac{\pi}{6}$ to $c(\hat{\Delta})$ as shown in Figure 3.2(xii). Observe that $d\left(v_{b^{-1}}\right)>3$ in $\hat{\Delta}$. If $d\left(v_{d^{-1}}\right)>2$ then $c(\hat{\Delta}) \leq-\frac{\pi}{3}$; or if $d\left(v_{d^{-1}}\right)=2$ (as shown) and either $d\left(v_{c^{-1}}\right)>3$ or $d\left(v_{e^{-1}}\right)>3$ then $c(\hat{\Delta}) \leq-\frac{\pi}{6}$; or if $d\left(v_{d^{-1}}\right)=2$ and $d\left(v_{c^{-1}}\right)=d\left(v_{e^{-1}}\right)=3$ then $l\left(v_{e^{-1}}\right)=e^{-1} a^{2}$ forces $d\left(v_{f^{-1}}\right)>4$ in $\hat{\Delta}$ and $d\left(v_{c}\right)>4$ in $\Delta$ so add $c(\Delta) \leq \frac{\pi}{15}$ to $c(\hat{\Delta}) \leq-\frac{\pi}{10}$ as shown by the dotted arrow in Figure 3.2(xii).

This completes the distribution of curvature from $\Delta$. Checking Figures 3.2(iv)-(xii) shows that $\hat{\Delta}$ is uniquely associated with $\Delta$ in each case and it follows from the above that $c(\hat{\Delta})+c(\Delta) \leq 0$.

Case 9: $a=e$ and $b=c$. There are five subcases: (i) $d\left(v_{a}\right)=d\left(v_{c}\right)=2$; (ii) $d\left(v_{a}\right)=$ 


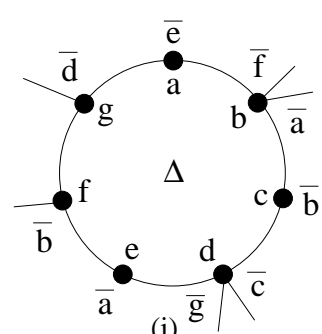

(i)

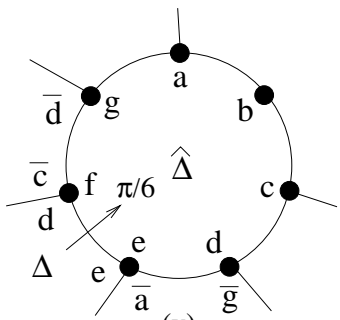

(v)

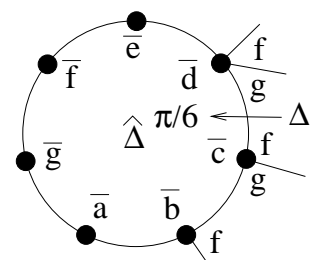

(ix)

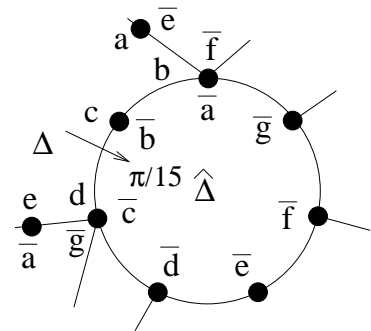

(ii)

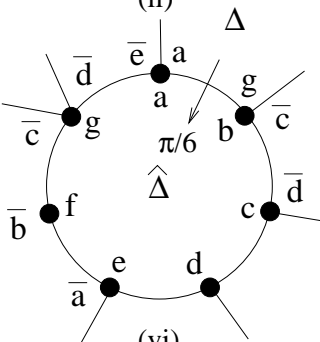

(vi)

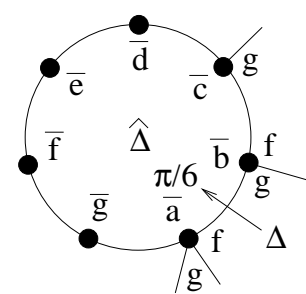

(x)

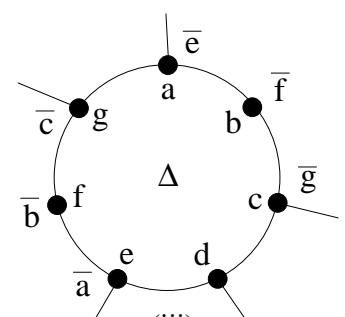

(iii)

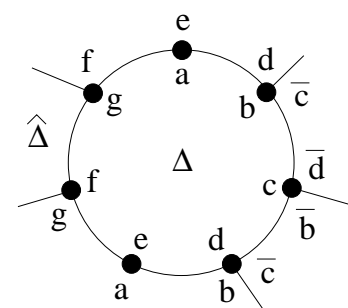

(vii)

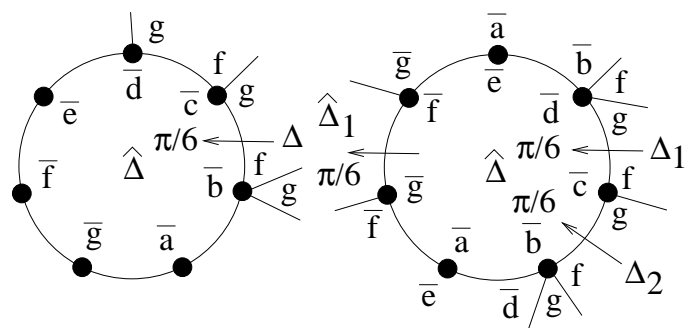

(xi) (iv)

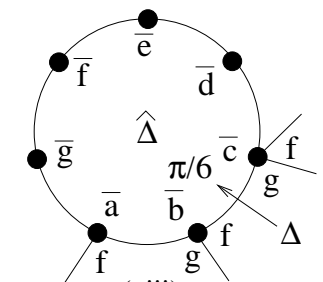

(xii)

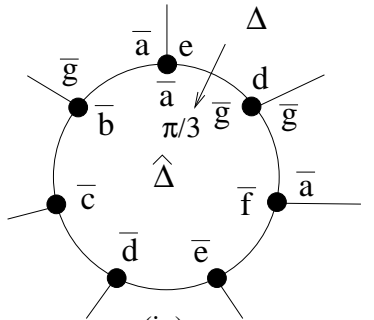

Figure 3.3: regions $\Delta$ and $\hat{\Delta}$ for Cases 9,3 and 2

$d\left(v_{e}\right)=2$; (iii) $d\left(v_{c}\right)=d\left(v_{e}\right)=2$; (iv) $d\left(v_{b}\right)=d\left(v_{e}\right)=2$; and (v) $d\left(v_{a}\right)=d\left(v_{c}\right)=d\left(v_{e}\right)=2$. In subcase (i) if $d\left(v_{g}\right)=d\left(v_{f}\right)=3$ then $l\left(v_{g}\right)=d^{2} g^{-1}$ and $l\left(v_{f}\right)=b^{-1} f a$ in which case $\mathcal{P}$ is aspherical by Lemma 2.2(iv); or if $d\left(v_{b}\right)=d\left(v_{c}\right)=d\left(v_{d}\right)=3$ then $l\left(v_{b}\right)=f^{-1} b\left\{a^{ \pm 1}, e^{ \pm 1}\right\}$. But if $l\left(v_{b}\right)=f^{-1} b\left\{a^{-1}, e^{-1}\right\}$ then Lemma 2.2(iv) applies so either $l\left(v_{b}\right)=f^{-1} b a, l\left(v_{c}\right)=$ $g c f^{-1}$ and $l\left(v_{d}\right)=d g^{-2}$ or $l\left(v_{b}\right)=f^{-1} b e, l\left(v_{c}\right)=d c f^{-1}$ and $l\left(v_{d}\right)=d g^{-2}$. But in each case $G$ is then cyclic. In subcase (ii) $l\left(v_{b}\right)=f^{-1} b a^{-1} w$ and $d\left(v_{b}\right)>3$ or Lemma 2.2(iv) applies; and $d\left(v_{d}\right)=3$ implies $l\left(v_{d}\right)=c^{-1} d f$ and then $l\left(v_{e}\right)=e^{2} w$ forcing $d\left(v_{e}\right)>3$. In subcase (iii) $l\left(v_{b}\right)=b a^{-1} w$ so either $d\left(v_{b}\right)>3$ or Lemma 2.2(iv) applies; and $l\left(v_{d}\right)=c^{-1} d g^{-1} w$ so $d\left(v_{d}\right)>3$. In subcase (iv) $l\left(v_{b}\right)=a b^{-1} w$ so either $d\left(v_{a}\right)>3$ or Lemma 2.2(iv) applies; and $d\left(v_{c}\right)=d\left(v_{d}\right)=d\left(v_{f}\right)=3$ forces $l\left(v_{c}\right)=d^{-1} c f^{-1}, l\left(v_{d}\right)=d g^{-2}$ and $l\left(v_{f}\right)=b^{-1} f\left\{a^{ \pm 1}, e^{ \pm 1}\right\}$ and $G$ is then cyclic. In subcases (i)-(iv) either $c(\Delta) \leq 0$ or $G$ is cyclic or Lemma 2.2(iv) applies. This leaves subcase (v) and $\Delta$ is shown in Figure 3.3(i) where observe that $d\left(v_{d}\right)>3$. If $d\left(v_{b}\right)=3$ then $l\left(v_{b}\right)=f^{-1} b a^{-1}$ which implies $a=f^{-1}$ and Lemma 2.2(iv) applies, therefore $d\left(v_{b}\right)>3$ also. If $d\left(v_{f}\right)>3$ and $d\left(v_{g}\right)>3$ then $c(\Delta) \leq 0$ so assume

otherwise. Let $d\left(v_{g}\right)=3$. Then $l\left(v_{g}\right)=g d^{-1}\left\{d^{-1}, g\right\}$. If $l\left(v_{g}\right)=g d^{-2}$ then $d\left(v_{f}\right)>$ 3 , indeed if $d\left(v_{f}\right)=4$ then $l\left(v_{f}\right)=b^{-1} f c^{-1} f$ and $f^{2}=1$, so $d\left(v_{f}\right)>4$. Moreover $d\left(v_{b}\right)=d\left(v_{d}\right)=4$ yields a contradiction and so $c(\Delta) \leq c(2,2,2,3,4,5,5)<0$. Let 
$l\left(v_{g}\right)=g^{2} d^{-1}$. If $d\left(v_{f}\right)=3$ then $a=f^{-1}$ and Lemma 2.2(iv) applies so $d\left(v_{f}\right)>3$. Now $d\left(v_{d}\right)=4$ forces $l\left(v_{d}\right)=c^{-1} d g^{-1} f$ and given this, $d\left(v_{b}\right)=4$ or $d\left(v_{f}\right)=4$ yields a contradiction, so $c(\Delta) \leq 0$. Therefore $d\left(v_{d}\right)>4$ and $c(\Delta) \leq c(2,2,2,3,4,4,5)=\frac{\pi}{15}$. Now let $d\left(v_{f}\right)=3$ and so $d\left(v_{g}\right)>3$. Then $l\left(v_{f}\right)=b^{-1} f\left\{a^{ \pm 1}, e^{ \pm 1}\right\}$. But $l\left(v_{f}\right)=b^{-1} f\{a, e\}$ implies $f=a^{-1}$ or $f=e^{-1}$ and Lemma 2.2(iv) applies; so $l\left(v_{f}\right)=b^{-1} f\left\{a^{-1}, e^{-1}\right\}$ and this forces $d\left(v_{b}\right)>4$ and $c(\Delta) \leq \frac{\pi}{15}$. In each case add $c(\Delta) \leq \frac{\pi}{15}$ to $c(\hat{\Delta})$ as shown in Figure 3.3(ii). Then $\hat{\Delta}$ is uniquely associated with $\Delta$ and, since either $d\left(v_{a^{-1}}\right)>4$ or $d\left(v_{c^{-1}}\right)>4$ in $\hat{\Delta}, c(\hat{\Delta})+c(\Delta) \leq c(2,2,3,3,3,4,5)+\frac{\pi}{15}<0$.

Case 4: $b=c$. In this subcase $d\left(v_{b}\right)=2$ forces $d\left(v_{c}\right)>2$ and so $c(\Delta) \leq 0$.

Case 6: $a=e^{-1}$ and $b=f$. There are four subcases: (i) $d\left(v_{a}\right)=d\left(v_{e}\right)=2$; (ii) $d\left(v_{a}\right)=d\left(v_{f}\right)=2$; (iii) $d\left(v_{b}\right)=d\left(v_{e}\right)=2$; (iv) $d\left(v_{b}\right)=d\left(v_{f}\right)=2$. But $d\left(v_{b}\right)=2$ forces $l\left(v_{a}\right)=a e^{-1} w$ and then $d\left(v_{a}\right)>3 ; d\left(v_{f}\right)=2$ forces $l\left(v_{e}\right)=e a^{-1} w$ and then $d\left(v_{e}\right)>3 ; d\left(v_{a}\right)=2$ forces $l\left(v_{b}\right)=d b w$ and $l\left(v_{g}\right)=g f w$ therefore $d\left(v_{b}\right)=d\left(v_{g}\right)=3$ will yield $d=c=g$ and $\mathcal{P}$ is aspherical by Lemma 2.1(i); and similarly if $d\left(v_{e}\right)=2$ then $d\left(v_{d}\right)=d\left(v_{f}\right)=3$ yields $d=g$ and again Lemma 2.1(i) applies. It follows that either $c(\Delta) \leq 0$ or $\mathcal{P}$ is aspherical by Lemma 2.1(i).

Case 7: $a=e^{-1}$ and $b=c$. There are five subcases: (i) $d\left(v_{a}\right)=d\left(v_{c}\right)=2$; (ii) $d\left(v_{a}\right)=$ $d\left(v_{e}\right)=2$; (iii) $d\left(v_{b}\right)=d\left(v_{e}\right)=2$; (iv) $d\left(v_{c}\right)=d\left(v_{e}\right)=2 ;$ (v) $d\left(v_{a}\right)=d\left(v_{c}\right)=d\left(v_{e}\right)=2$. But if $d\left(v_{a}\right)=2$ and $d\left(v_{g}\right)=3$ then $l\left(v_{g}\right)=g f\left\{b^{-1}, c^{-1}\right\}$ and $\mathcal{P}$ is aspherical by Lemma $2.1(\mathrm{vi})$; similarly $d\left(v_{e}\right)=2$ forces $d\left(v_{f}\right)>3$; if $d\left(v_{a}\right)=d\left(v_{c}\right)=2$ then $l\left(v_{b}\right)=g b a^{-1} w$ and $d\left(v_{b}\right)>3$; and if $d\left(v_{c}\right)=d\left(v_{e}\right)=2$ then $l\left(v_{d}\right)=c^{-1} d b w$ and $d\left(v_{d}\right)>3$. It follows that for subcases (i), (ii), (iv) and (v) either $c(\Delta) \leq 0$ or $\mathcal{P}$ is aspherical by Lemma 2.1(vi). For subcase (iii) $d\left(v_{e}\right)=2$ forces $d\left(v_{f}\right)>3$ as above and if $d\left(v_{c}\right)=3$ then $l\left(v_{c}\right)=d^{-1} c f^{-1}$ forcing $l\left(v_{d}\right)=c^{-1} d b w$ therefore $d\left(v_{d}\right)>3$ and $c(\Delta) \leq 0$.

In Case 10, $s_{2}(t)=a t^{3} d t^{-1} e t^{2} g t^{-1}=1$ is solvable by Theorem 1 in [13].

For the final remaining cases let $c^{*}(\hat{\Delta})$ denote $c(\hat{\Delta})$ plus all the positive curvature $\hat{\Delta}$ receives from other regions minus the amount of curvature $\hat{\Delta}$ gives to other regions as a result of the curvature distribution that takes place. We show that $c^{*}(\hat{\Delta}) \leq 0$. Since $c(K) \leq \sum c^{*}(\hat{\Delta})$ where the sum is taken over all $\hat{\Delta}$ that receives positive curvature such that $c^{*}(\hat{\Delta})>0$, it follows that $c(K) \leq 0$, a contradiction to $c(K)=4 \pi$.

Case 3: $b=f$. Here $c(\Delta)>0$ implies $l\left(v_{b}\right)=b f^{-1}, l\left(v_{f}\right)=f b^{-1}$ and $\Delta$ is given by Figure 3.3(iii). Suppose that $d\left(v_{c}\right)=d\left(v_{d}\right)=d\left(v_{e}\right)=3$. Then $l\left(v_{e}\right)=e a^{-1}\left\{a^{-1}, e\right\}$. Let $l\left(v_{e}\right)=e a^{-2}$. Since $l\left(v_{d}\right)=d^{2} g^{-1}$ implies $d\left(v_{c}\right)>3$ we must have $l\left(v_{d}\right)=d g^{-2}$ forcing $l\left(v_{c}\right)=g^{-1} c f^{-1}$. Add $c(\Delta) \leq \frac{\pi}{3}$ to $c(\hat{\Delta})$ as shown in Figure 3.3(iv) in which, observe, if $l\left(v_{f^{-1}}\right)=a^{-1} f^{-1}\{b, c\}$ there is a contradiction, so $d\left(v_{f^{-1}}\right)>3$ and $c(\hat{\Delta}) \leq-\frac{\pi}{2}$. Now let $l\left(v_{e}\right)=e^{2} a^{-1}$. Then $l\left(v_{d}\right)=d f c^{-1}$ forcing $l\left(v_{c}\right)=c b^{-1} g^{-1}$; and $l\left(v_{a}\right)=a e^{-2}$ which forces $d\left(v_{g}\right)>3$. Add $c(\Delta) \leq \frac{\pi}{6}$ to $c(\hat{\Delta})$ as shown in Figure 3.3(v). Note that if $d\left(v_{d}\right)=3$ or $d\left(v_{g}\right)=3$ in $\hat{\Delta}$ of Figure 3.3(v) there is a contradiction and so $c(\hat{\Delta}) \leq-\frac{\pi}{3}$. This completes the subcase $d\left(v_{c}\right)=d\left(v_{d}\right)=d\left(v_{e}\right)=3$. If $d\left(v_{c}\right)>3$ or $d\left(v_{d}\right)>3$ or $d\left(v_{e}\right)>3$ in $\Delta$ then 
$c(\Delta)>0$ implies $d\left(v_{a}\right)=d\left(v_{g}\right)=3$ which forces $l\left(v_{a}\right)=a^{2} e^{-1}$ and $l\left(v_{g}\right)=g b c^{-1}$. Add $c(\Delta) \leq \frac{\pi}{6}$ to $c(\hat{\Delta})$ as shown in Figure 3.3(vi). Observe that if $d\left(v_{f}\right)>2$ in $\hat{\Delta}$ in Figure $3.3(\mathrm{vi})$ then $c(\hat{\Delta}) \leq-\frac{\pi}{3}$, or if $d\left(v_{f}\right)=2$ as shown then $d\left(v_{g}\right)>3$ and $c(\hat{\Delta}) \leq-\frac{\pi}{6}$. This completes the distribution of curvature. It follows that if $\hat{\Delta}$ receives positive curvature from exactly one region $\Delta$ then $c^{*}(\hat{\Delta})=c(\hat{\Delta})+c(\Delta) \leq 0$. If $\hat{\Delta}$ receives from more than one region then we see from Figure 3.3(iv)-(vi) that $\hat{\Delta}$ is given by Figure 3.3(iv) and by Figure $3.3(\mathrm{vi})$ (in which it is assumed that $d\left(v_{f}\right)>2$ ) and it follows that $c^{*}(\hat{\Delta}) \leq-\frac{\pi}{2}+\frac{\pi}{3}+\frac{\pi}{6}=0$.

This leaves Case 2: $a=e^{-1}$. Here $c(\Delta)>0$ implies $l\left(v_{a}\right)=l\left(v_{e}\right)=a e$. If now $d\left(v_{f}\right)=3$ then $l\left(v_{f}\right)=g f\left\{b^{-1}, c^{-1}\right\}$ forcing $d\left(v_{g}\right)>3$. Since $c(2,2,3,3,3,4,4)=0$ it follows that either $d\left(v_{f}\right)=3$ or $d\left(v_{g}\right)=3$ and $d\left(v_{b}\right)=d\left(v_{c}\right)=d\left(v_{d}\right)=3$. Therefore $\Delta$ is given by Figure 3.3(vii). If $d\left(v_{f}\right)=3$ then distribute $c(\Delta) \leq \frac{\pi}{6}$ to $c(\hat{\Delta})$ as shown in Figures 3.3(viii) and (ix); and if $d\left(v_{g}\right)=3$ then add $c(\Delta) \leq \frac{\pi}{6}$ to $c(\hat{\Delta})$ as shown in Figures 3.3(x) and (xi).

Consider $\hat{\Delta}$ of Figure 3.3(viii). If $d\left(v_{a^{-1}}\right)=3$ then $l\left(v_{a^{-1}}\right)=f a^{-1}\left\{b^{-1}, c^{-1}\right\}$ and this forces $d\left(v_{g^{-1}}\right)>3$, so $c(\hat{\Delta}) \leq c(2,3,3,3,3,4,4)=-\frac{\pi}{3}$. For $\hat{\Delta}$ of Figure 3.3(ix) if $d\left(v_{a^{-1}}\right)>2$ or $d\left(v_{e^{-1}}\right)>2$ then $c(\hat{\Delta}) \leq-\frac{\pi}{6}$; or if $d\left(v_{a^{-1}}\right)=d\left(v_{e^{-1}}\right)=2$ then $d\left(v_{b^{-1}}\right)>3$ and, as in Figure 3.3 (vii), either $d\left(v_{f^{-1}}\right)>3$ or $d\left(v_{g^{-1}}\right)>3$ and $c(\hat{\Delta}) \leq-\frac{\pi}{6}$. In Figure $3.3(\mathrm{x}), c(\hat{\Delta}) \leq-\frac{\pi}{6}$. For $\hat{\Delta}$ of Figure 3.3(xi) if $l\left(v_{e^{-1}}\right)=e^{-1} a^{-1}$ then $d\left(v_{d^{-1}}\right)>3$ and it follows that $c(\hat{\Delta}) \leq-\frac{\pi}{6}$.

Let $\hat{\Delta}$ be a region that receives positive curvature in the distribution described above. If $\hat{\Delta}$ receives from exactly one neighbour then $c^{*}(\hat{\Delta}) \leq-\frac{\pi}{6}+\frac{\pi}{6}=0$. An inspection of Figure 3.3(viii)-(xi) shows that the only other possibility is that $\hat{\Delta}$ receives from exactly two neighbours $\Delta_{1}$ and $\Delta_{2}$ say, and there are two cases. The first case is when $\Delta_{1}=\Delta$ of Figure 3.3(viii) and $\Delta_{2}=\Delta$ of Figure 3.3(x). But then $c^{*}(\hat{\Delta})=c(\hat{\Delta})+c\left(\Delta_{1}\right)+c\left(\Delta_{2}\right) \leq$ $-\frac{\pi}{3}+2\left(\frac{\pi}{6}\right)=0$ and note that $g f b^{-1}=1$ in $G$ for this case. The only other possibility is $\Delta_{1}=\Delta$ of Figure 3.3(ix) and $\Delta_{2}=\Delta$ of Figure 3.3(xi) and here $g f c^{-1}=1$ in $G$. If $c(\hat{\Delta}) \leq-\frac{\pi}{3}$ then $c^{*}(\hat{\Delta}) \leq 0$ so suppose otherwise. Then $d\left(v_{a^{-1}}\right)=d\left(v_{e^{-1}}\right)=2$ and $\hat{\Delta}, \Delta_{1}$ and $\Delta_{2}$ are given by Figure 3.3(xii) where we note that if $d\left(v_{g}\right) \geq 6$ in $\Delta_{1}$ then $c\left(\Delta_{1}\right) \leq 0$ and $d\left(v_{g}\right)=5$ yields a contradiction, so $d\left(v_{g}\right)=4$ as shown and the same argument applies to $v_{f}$ of $\Delta_{2}$. Distribute $c^{*}(\hat{\Delta}) \leq \frac{\pi}{6}$ to $c\left(\hat{\Delta}_{1}\right)$ as shown and note that, as a result of this, $c^{*}(\hat{\Delta})$ is then at most $\frac{\pi}{6}-\frac{\pi}{6}=0$.

Now repeat the above argument with $\hat{\Delta}_{1}$ of Figure 3.3 (xii) playing the rôle of $\hat{\Delta}$ of Figure 3.3(vii). Again either $c^{*}\left(\hat{\Delta}_{1}\right) \leq 0$ or $c\left(\hat{\Delta}_{1}\right)=-\frac{\pi}{6}$ and $\hat{\Delta}_{1}$ receives positive curvature from exactly two neighbours. The key points to note is that positive curvature is always distributed at each stage across $(b, c)^{ \pm 1}$ or $(c, d)^{ \pm 1}$ edges and that the region receiving positive curvature is always itself negatively curved. It follows that the graph obtained from the union of distribution paths so constructed contains no cycles and so this procedure must each time terminate at a region $\hat{\Delta}_{k}$, say, for which $c^{*}\left(\hat{\Delta}_{k}\right) \leq 0$. This implies that the total curvature of $K$ is non-positive, a contradiction, which completes the proof under the assumption $l\left(v_{0}\right)=1$ in $G$.

Now assume that $l\left(v_{0}\right) \neq 1$ in $G$ and let $d\left(v_{0}\right)=k_{0}$. A region of $K$ is interior if it does 
not involve $v_{0}$; otherwise it is said to be a boundary region. For each interior region $\Delta$ such that $c(\Delta)>0$ distribute $c(\Delta)$ to the region $\hat{\Delta}$ exactly as before as shown in Figures 3.1-3.3 except possibly Figure 3.3(xii) of Case 2. As described above this formed the start of a sequence of distributions that terminated at a region $\hat{\Delta}_{k}$ for which $c^{*}\left(\hat{\Delta}_{k}\right) \leq 0$. The difference here is if there is a $\hat{\Delta}_{j}$ where $1 \leq j \leq k$ which is a boundary region then the distribution terminates at the first such region encountered and the $2 \cdot \frac{\pi}{6}$ remains with $\hat{\Delta}_{j}$. We have shown that if $\hat{\Delta}$ is always interior then $c^{*}(\hat{\Delta}) \leq 0$ and so it follows that $c(K) \leq \sum c^{*}(\hat{\Delta})$ where the sum is taken over all the boundary regions of $K$.

Let $\hat{\Delta}$ be a boundary region of $K$. Checking Figures 3.1, 3.2 and 3.3(i), (ii) shows that $\hat{\Delta}$ remains uniquely associated with $\Delta$ and so the maximum curvature $\hat{\Delta}$ can receive is $\frac{\pi}{3}$; for Case 3 the maximum $\hat{\Delta}$ can received is $\frac{\pi}{3}+\frac{\pi}{6}$ (see Figure 3.3(iv)-(vi)); and for Case 2 the maximum $\hat{\Delta}$ can receive is $2 \cdot \frac{\pi}{6}$ (see Figure 3.3 (viii)-(xii)). Suppose that $\hat{\Delta}$ has at most one interior $\left(\neq v_{0}\right)$ vertex of degree 2. Then $c^{*}(\hat{\Delta}) \leq c\left(k_{0}, 2,3,3,3,3,3\right)+\left(\frac{\pi}{3}+\frac{\pi}{6}\right)=\frac{2 \pi}{k_{0}}-\frac{\pi}{6}$. (Note that this clearly holds if $k_{0} \geq 3$; and if $k_{0}=2$ then at most one vertex of $\hat{\Delta}$ can coincide with $v_{0}$.) Now let $\hat{\Delta}$ have exactly two interior vertices of degree 2 . Then $\hat{\Delta}$ cannot be the region $\hat{\Delta}$ of Figure 3.3(iv)-(vi) and so $c^{*}(\hat{\Delta}) \leq c\left(k_{0}, 2,2,3,3,3,3\right)+\frac{\pi}{3}=\frac{2 \pi}{k_{0}}$. Finally suppose that $\hat{\Delta}$ has the maximum of three interior vertices of degree 2 . Then either $c^{*}(\hat{\Delta})=c(\hat{\Delta}) \leq c\left(k_{0}, 2,2,2,3,3,3\right)=\frac{2 \pi}{k_{0}}$ or $\hat{\Delta}$ is given by Figure 3.2(iv), in which case $c^{*}(\hat{\Delta}) \leq c\left(k_{0}, 2,2,2,3,5,6\right)+\frac{\pi}{15}=\frac{2 \pi}{k_{0}}-\frac{3 \pi}{5}$. In conclusion it follows that $c^{*}(\hat{\Delta}) \leq \frac{2 \pi}{k_{0}}$ for any given boundary region $\hat{\Delta}$ and so $c(K) \leq k_{0} \cdot\left(\frac{2 \pi}{k_{0}}\right)<4 \pi$, our final contradiction.

\section{References}

[1] W. A. Bogley and S. J. Pride, Aspherical relative presentations, Proc. Edinburgh Math. Soc. 35 (1992) 1-39.

[2] S. D. Brodskii and J. Howie, One-relator products of torsion-free groups, Glasgow Math. J. 35 (1993) 99-104.

[3] A. S. Clark, Disk diagrams, tessellations of the 2-sphere, and equations over torsionfree groups, Comm. Algebra 39 (2011), no. 8, 2981-3020.

[4] A. Clifford and R. Z. Goldstein, Tesselations of $S^{2}$ and equations over torsion-free groups, Proc. Edinburgh Math. Soc. (2) 38 (1995), no. 3, 485-493.

[5] A. Clifford and R. Z. Goldstein, Equations with torsion-free coefficients, Proc. Edinburgh Math. Soc. (2) 43 (2000), no. 2, 295-307.

[6] A. Clifford and R. Z. Goldstein, The group $\langle G, t \mid e\rangle$ when $G$ is torsion-free, J Algebra 245 (2001), no. 1, 297-309. 
[7] M. Edjvet, Equations over groups and a theorem of Higman, Neumann and Neumann, Proc. London Math. Soc. 62 (1991) 563-589.

[8] R. Fenn, C. Rourke, Klyachko's methods and the solution of equations over torsionfree groups, Enseign. Math. (2) 42 (1996), no. 1-2, 49-74.

[9] R. Fenn, C. Rourke, Characterisation of a class of equations with solutions over torsion-free groups, The Epstein birthday schrift, 159-166 (electronic), Geom. Topol. Monogr. 1, Geom. Topol. Publ., Coventry, 1998.

[10] J. Howie, On pairs of 2-complexes and systems of equations over groups, J. Reine Angew. Math. 324 (1981), 165-174.

[11] J. Howie, The solution of length three equations over groups, Proc. Edinburgh Math. Soc. (2) 26 (1983), 89-96.

[12] S. V. Ivanov and A. A. Klyachko, Solving equations of length at most six over torsionfree groups, J. Group Theory 3 (2000), no. 3, 329-337.

[13] S. K. Kim, Some equations over torsion-free groups, Internat. J. Algebra Comput. 16 (2006), no. 2, 369-376.

[14] A. A. Klyachko, A funny property of a sphere and equations over groups, Comm. Algebra 21 (1993), 2555-2575.

[15] F. Levin, Solutions of equations over groups, Bull. Amer. Math. Soc. 68 (1962), 603-604.

[16] M. I. Prishchepov, On small length equations over torsion-free groups, Internat. J. Algebra Comput. 4 (1994), no. 4, 575-589.

[17] J. R. Stallings, A graph-theoretic lemma and group embeddings, Ann. Math. Stud. 111 (1987) 145-155. 